\title{
Avaliação de serviços de saúde mental: adaptação transcultural de uma medida da percepção dos usuários sobre os resultados do tratamento
}

\author{
Mental health services evaluation: transcultural \\ adaptation of a user's reported outcome measure \\ Marina Bandeira', Maria Gláucia Pires Calzavara', Cecília Silva Costa², Luciana Cesari
}

\section{RESUMO}

Introdução: Para avaliar a qualidade dos serviços de saúde mental, medidas de resultados relatados pelos próprios usuários têm sido destacadas e, mais recentemente, a percepção de mudanças. O objetivo desta pesquisa foi realizar a adaptação transcultural para o Brasil da escala canadense Questionnaire of Perceived Change, obtendo-se a Escala de Mudança Percebida (EMP), em duas versões: a dos pacientes e a dos familiares. Método: Participaram da pesquisa 20 familiares e 23 pacientes psiquiátricos de um serviço de saúde mental de uma cidade de porte médio de Minas Gerais. A escala foi submetida aos procedimentos de adaptação transcultural: tradução, retradução, análise por comissão de especialistas, estudo piloto. A escala original possui 20 itens que avaliam a mudança percebida em quatro dimensões da vida dos pacientes. As alternativas de resposta estão dispostas em escala tipo Likert de 4 pontos. Resultados: Foram feitas modificações, incluindo: uma nova forma de aplicação por um entrevistador, número balanceado de alternativas de resposta resultando em três opções, redação dos itens em linguagem simples, eliminação de um item, uso de exemplos e inclusão de duas perguntas abertas. A escala mostrou-se de fácil compreensão pelos usuários. Conclusão: As duas versões da escala EMP estão adaptadas ao contexto brasileiro e apresentam equivalência semântica com a escala original. Elas servirão para avaliar os resultados do tratamento, na percepção dos seus usuários.

\section{ABSTRACT}

Introduction: To evaluate the quality of mental health services, patient-reported outcome measures have been used and, more recently, a measure of perception of change by users. This research aimed to make the transcultural adaptation to Brazil of the canadian Questionnaire of Perceived Changes, in order to obtain the Escala de Mudança Percebida (EMP), in two forms, patient-scale and family-scale. Method: Twenty family members and 23 psychiatric patients from a mental health services in a median size town of Minas Gerais, Brazil, participated in this research.

1 Universidade Federal de São João Del Rei (UFSJ).

2 Universidade Federal de São João Del Rei (UFSJ), Departamento de Psicologia. 


\section{Keywords}

Patient-reported outcome, perception of change, mental health services, scale.
The scale was submitted to transcultural adaptation procedures: translation, backtranslation, analysis by a specialists committee and pilot study. The original scale is composed of 20 items which evaluate the users' perceived change in four domains of patient's life. Response alternatives are disposed in a four point Likert scale. Results: The scale was modified, including: a new application procedure by an interviewer, a balanced number of response alternatives with three options, the elimination of one item, a more simple elaboration of items, the use of examples and the inclusion of two open questions. The scales were easily understood by users. Conclusion: The two EMP scale forms are adapted to Brazilian context and have semantic equivalence to the original scale. They will be useful to evaluate the treatment results according to the users' perception.

\section{INTRODUÇÃO}

A avaliação de serviços de saúde mental deve ser uma atividade contínua, realizada periodicamente, a fim de identificar aspectos da assistência aos pacientes que necessitam ser reajustados e promover a melhoria do tratamento oferecido e a qualidade dos serviços'. Entre as dez recomendações da Organização Mundial da Saúde para a saúde mental, encontra-se o desenvolvimento da pesquisa e do monitoramento dos serviços, com indicadores da qualidade do acesso, da adequação da assistência, da qualidade das intervenções preventivas e terapêuticas e da avaliação dos resultados do tratamento etc.!

A tríade estrutura, processo e resultados, proposta por Donabedian², tem sido destacada na literatura para a avaliação dos serviços de saúde. Os resultados do tratamento têm recebido especial atenção dos pesquisadores e são tradicionalmente avaliados com medidas objetivas sobre o estado clínico do paciente, antes e depois do tratamento, idealmente em delineamentos de pesquisa com controle de vieses, para maior validade interna. Medidas objetivas indiretas são também utilizadas, antes e após o tratamento, tais como a frequência e a duração das internações ocorridas, a frequência de utilização das urgências psiquiátricas, o aumento no nível de funcionalidade e a autonomia dos pacientes, a qualidade de vida, entre outras ${ }^{3}$.

Uma tendência que surgiu nas últimas três décadas se refere à inclusão dos usuários na avaliação dos serviços e, em consequência, à utilização de medidas subjetivas de autorrelatos dos pacientes sobre a percepção deles a respeito dos serviços e dos resultados do tratamento ${ }^{3-6}$. A avaliação por pacientes e por seus familiares tem papel importante na promoção da qualidade dos serviços, pois fornece informações pertinentes a respeito tanto da relação interpessoal no atendimento quanto dos resultados do tratamento. Segundo Donabedian? ${ }^{7}$ a qualidade técnica da assistência em saúde depende não apenas do que é feito, mas do resultado obtido, e este deve estar congruente com a perspectiva dos usuários, caso contrário a qualidade do tratamento deixa a desejar. As medidas subjetivas do tratamento têm tido interesse crescente por parte dos clínicos, dos administradores e das agências de credenciamento de serviços ${ }^{8}$. Tem sido recomendado que a avaliação e o desenvolvimento dos serviços de saúde mental devem ser integrativos, incluindo a participação dos três agentes envolvidos nos serviços: usuários, familiares e profissionais do serviço'. Hansson ${ }^{9}$ apresenta sete recomendações para a avaliação de serviços de saúde mental e, entre elas, o uso de múltiplas perspectivas de avaliação, incluindo, além dos três agentes citados anteriormente, medidas de observadores independentes externos aos serviços, assim como o uso concomitante de medidas objetivas e subjetivas, pois as medidas subjetivas estão mais relacionadas com a utilização dos serviços. A avaliação dos resultados relatados pelos pacientes tem sido cada vez mais utilizada, em consequência de uma crescente visão dos pacientes como participantes ativos em seu tratamento e a partir da aceitação de que não só os resultados clínicos objetivos são importantes, mas também a percepção subjetiva dos pacientes 5 .

As medidas subjetivas dos resultados do tratamento ou critérios subjetivos de avaliação $0^{5}$ têm sido ainda denominadas como "resultados relatados pelos próprios pacientes" (patient-reported outcomes), também conhecidas como "instrumentos PRO", e têm sido definidas como "qualquer medida baseada na percepção e no julgamento do paciente sobre a sua doença e seu tratamento, medido diretamente por ele, sem a aferição do clínico ou do pesquisador, avaliando como a pessoa funciona ou como ela se sente em relação ao seu estado de saúde ${ }^{\prime \prime, 5}$. Os instrumentos PRO podem envolver a medida de conceitos dos mais simples (sintomas específicos) aos mais complexos (por exemplo, autonomia nas atividades cotidianas, qualidade de vida), assim como conceitos observáveis (por exemplo, coordenação motora) ou privados (por exemplo, sentir-se deprimido), mas devem ser submetidos a procedimentos metodológicos que comprovem sua fidedignidade e validade para medir o conceito que visa avaliar.

Diversos argumentos têm sido utilizados para defender a inclusão de medidas subjetivas na avaliação dos resultados do tratamento em serviços de saúde mental. Tem-se desta- 
cado que esse tipo de avaliação é importante porque fornece informações que não estão presentes nas medidas clínicas dos profissionais sobre os efeitos do tratamento, como mudanças nos eventos privados (por exemplo, sintomas, sentimentos, disposição), no funcionamento do paciente e no seu bem-estar, tal como percebido por ele $e^{5}$. Hasler et al. ${ }^{10}$ destacaram a importância das medidas subjetivas dos resultados, pois nem sempre há correspondência entre o que os usuários percebem como mudança desejável e os resultados objetivos obtidos com o tratamento. Pequenas mudanças produzidas pelo tratamento podem fazer grande diferença para os usuários, enquanto mudanças significativas em medidas clínicas objetivas podem ser consideradas irrelevantes na percepção dos usuários. Mercier et al. ${ }^{3}$ destacaram a necessidade de se aferir o impacto real de mudanças produzidas pelo tratamento na vida dos pacientes, percebidas por ele, o que serviria para calibrar as medidas objetivas de resultados do tratamento e constituir um indicador da validade social das intervenções efetuadas.

A inclusão da perspectiva dos pacientes pode também contribuir para aumentar a satisfação deles com o tratamento, o que tem sido associado com uma melhor adesão e utilização dos serviços, diminuindo a taxa de abandono $0^{9-11}$, além de ser um fator preditivo para menor taxa de hospitalizações futuras ${ }^{12}$. Esse aspecto da adesão ganha importância particular quando se consideram as altas taxas de abandono de tratamento em serviços de saúde mental, relatadas na literatura ${ }^{1,13}$. Tait e Lester ${ }^{14}$ destacam essa e diversas outras vantagens na inclusão da perspectiva dos pacientes. Por exemplo, o paciente tem conhecimento privilegiado da vivência de uma doença mental, dos seus sintomas, das suas necessidades e da experiência com os serviços de saúde mental, que pode ser usado para aprimorar o tratamento e aumentar o conhecimento dos profissionais da área sobre a doença mental. Eles possuem uma perspectiva particular, diferente da visão dos profissionais, e as medidas subjetivas podem servir para mostrar as prioridades deles, redirecionando as características do cuidado prestado pelo serviço. Além disso, a inclusão dos pacientes na avaliação pode ter efeito terapêutico, pois pode aumentar a autoestima e o sentimento de controle deles, o que aumenta o empoderamento (empowerment), ao perceberem que o ponto de vista deles está sendo considerado. Nesse sentido, incluir a perspectiva dos usuários não só fornece informações sobre os resultados do tratamento como também afeta o próprio tratamento ${ }^{14}$.

Observa-se na literatura pouca referência à inclusão de medidas subjetivas da percepção dos familiares de pacientes psiquiátricos, embora seja igualmente importante na avaliação de resultados, em particular quando os familiares atuam como cuidadores. Essa é uma informação privilegiada, pois se trata do principal provedor de cuidados cotidianos aos pacientes, com uma visão muito próxima de seus comportamentos, nível de funcionamento e das mudanças que ocorrem em sua vida, em sua rotina, em sua autonomia, em função do tratamento recebido. Além disso, incluir a perspectiva dos familiares na avaliação dos resultados pode ainda ter um efeito positivo na satisfação deles com o serviço e na diminuição da sobrecarga deles ${ }^{15,16}$. Apesar de ser menos utilizada, a inclusão da perspectiva dos familiares tem sido recomendada, na avaliação e no desenvolvimento dos serviços ${ }^{1,9}$. Porém, essa avaliação tem se limitado a medidas de satisfação com o serviço e a medidas de sobrecarga com o papel de cuidador.

Dentre os instrumentos de medida desenvolvidos e validados para avaliar a percepção dos pacientes sobre os resultados do tratamento, destacam-se dois grupos de escalas: as que avaliam o bem-estar psicológico e as que avaliam os resultados do tratamento 5 . Neste segundo grupo, pertinente ao presente artigo, as escalas de medida avaliam: grau de satisfação com os serviços, necessidades percebidas de tratamento, relação terapêutica, sintomas, conhecimento sobre a doença e sobre o tratamento, efeitos colaterais, qualidade da comunicação com o terapeuta ${ }^{5,8}$ e mudanças percebidas ${ }^{3}$. Entre esses instrumentos, as medidas de satisfação têm sido as mais utilizadas, porém elas são elaboradas para avaliar um conjunto das dimensões dos serviços, tais como a estrutura do serviço (conforto, limpeza, entre outros), a forma de atendimento, a informação recebida sobre a doença e o tratamento, a acessibilidade, a privacidade etc. Essas medidas não enfatizam os efeitos ou resultados do tratamento, pois incluem apenas alguns poucos itens que avaliam especificamente esse aspecto ${ }^{17,18}$.

Uma escala de medida que visa avaliar especificamente os resultados do tratamento, em termos das mudanças percebidas pelos pacientes, em diversas dimensões da vida deles, foi elaborada recentemente por Mercier et al. ${ }^{3}$, no Canadá. Essa variável é importante porque interfere na relação entre resultados objetivos do tratamento e o grau de satisfação dos usuários, pois, se as mudanças objetivas, aferidas pelos profissionais, não forem percebidas pelos usuários, elas não terão efeito na satisfação deles, o que explicaria as baixas correlações encontradas entre essas medidas ${ }^{10}$. A medida de mudança percebida deve ser, portanto, de interesse do pesquisador que pretende investigar as relações entre todas essas variáveis e compreender em que condições os resultados do tratamento podem resultar em satisfação dos usuários.

O objetivo do presente trabalho foi fazer a adaptação transcultural para o Brasil da escala de mudanças percebidas pelos pacientes ${ }^{3}$. Além disso, foi elaborada, no presente trabalho, uma segunda escala inédita dirigida aos familiares, com base na escala dos pacientes, tendo em vista a importância da percepção dos familiares como cuidadores que participam do cotidiano dos pacientes. Este artigo é o resultado da primeira parte de um projeto de pesquisa 
mais amplo, que visa à validação desses dois instrumentos de medida e que está atualmente em andamento no Laboratório de Pesquisa em Saúde Mental da UFSJ.

\section{MÉTODO}

\section{Descrição do instrumento de medida original}

A escala original, a partir da qual se baseou este trabalho, consiste no Questionnaire of Perceived Changes, elaborado por Mercier et al. ${ }^{3}$, no Canadá, para avaliar a percepção de pacientes psiquiátricos sobre os resultados do tratamento recebido em serviços de saúde mental. Não há uma versão original dirigida aos familiares. A versão brasileira dessa escala para familiares é, portanto, inédita.

A escala original possui 20 itens que avaliam o tipo de mudança percebida, nas seguintes dimensões da vida dos pacientes: 1) dimensão psicológica, 2) saúde física, 3) vida social e 4) condições de vida. Para cada item da escala, referente a um aspecto da vida do paciente, este é solicitado a relatar se percebeu alguma mudança desde que começou a ser tratado naquele serviço. As alternativas de resposta, para cada item da escala, se distribuem em uma escala tipo Likert de quatro pontos, sendo 1 pior do que antes, 2 sem mudança, 3 um pouco melhor do que antes e 4 muito meIhor do que antes. O último item da escala avalia como o paciente se sente, em geral, depois que iniciou o tratamento naquela instituição.

A primeira dimensão da escala é composta de seis itens que avaliam a percepção do paciente na dimensão psicológica, no que concerne aos seguintes aspectos: problemas pessoais, humor, estabilidade das emoções, confiança em si mesmo, interesse pela vida e capacidade de suportar situações difíceis. A segunda dimensão inclui cinco itens relacionados à saúde física do paciente, que avaliam as mudanças ocorridas no tocante a apetite, energia, sono, aspectos físicos e sexualidade. A terceira dimensão da escala refere-se à vida social do paciente e consiste de quatro itens que avaliam as mudanças percebidas por ele, relativamente aos seguintes aspectos: convivência com sua família, relacionamento com os amigos, convivência com outras pessoas, bem como seu interesse em trabalhar ou se ocupar com alguma coisa. Finalmente, a quarta dimensão da escala, referente às condições de vida, é composta por quatro itens que avaliam as mudanças percebidas pelo paciente relativamente a atividades de lazer, tarefas domésticas (por exemplo, cozinhar, fazer compras para a casa, lavar roupa, arrumar o quarto ou a casa etc.), capacidade de cumprir obrigações e tomar decisões e à situação financeira do paciente.

A escala original possui ainda uma questão aberta, feita no início da entrevista, com a qual se pergunta ao paciente se o tratamento que ele está recebendo naquela institui- ção o está ajudando a sentir-se melhor, cujas alternativas de resposta são do tipo "sim" ou "não". Em caso afirmativo, solicita-se que ele explique de que maneira o tratamento o fez sentir-se melhor. No caso de a resposta ser negativa, pede-se que ele explique por que o tratamento não o fez sentir-se melhor.

\section{Procedimento}

Antes de iniciar a pesquisa, obteve-se a autorização de um dos autores da escala para que ela fosse traduzida e adaptada para o Brasil. Após aprovação do projeto pela instituição psiquiátrica envolvida neste estudo e pela Comissão de Ética da UFSJ, os pacientes e familiares foram contatados no Centro de Atenção Psicossocial. As entrevistas para a coleta de dados foram realizadas individualmente, no momento em que os pacientes e familiares se encontravam no serviço para consultas. As entrevistas foram realizadas por estudantes de Psicologia, previamente treinados pelo primeiro autor, a fim de se obter uma padronização dos procedimentos de aplicação da escala, embora esta não necessite de uma formação clínica especial para ser aplicada Os sujeitos eram informados sobre os objetivos da entrevista, sobre o fato de que haveria sigilo a respeito dos dados e de que aquela entrevista não interferiria no tratamento que eles estavam recebendo e, em seguida, assinavam um termo de consentimento informado. Solicitava-se aos sujeitos que eles respondessem, de forma honesta, para cada aspecto de sua vida, se eles haviam melhorado, piorado ou estavam sem mudanças desde que haviam começado o tratamento naquela instituição, deixando-se claro que não havia respostas certas ou erradas.

A pesquisa foi realizada em duas etapas consecutivas, iniciando-se com a adaptação transcultural da escala de mudanças percebidas, versão dos pacientes (EMP-pacientes), seguida da elaboração e da análise da versão dos familiares (EMP-familiares).

\section{Procedimento de adaptação}

A adaptação transcultural, para o contexto brasileiro, da escala EMP-pacientes foi realizada seguindo os procedimentos recomendados pela literatura internacional da área $a^{19,20}$ (passos 1 a 5). Foi utilizada a versão em francês da escala original para a adaptação para o Brasil, em razão de a equipe de trabalho ter maior facilidade para lidar com essa versão. Após terminada a adaptação da escala EMP-pacientes, foi feita a elaboração da escala EMP-familiares, seguindo-se os passos 4 e 5 descritos a seguir.

1. Tradução: Inicialmente, um tradutor bilíngue, cuja língua materna era o português, traduziu a escala do francês para o português.

2. Retradução: Em seguida, um segundo tradutor bilíngue, cuja língua materna era o francês, fez a retradução, vertendo para o francês. 
3. Comparação das duas versões: As duas formas em francês, a original e a re-traduzida, foram então comparadas, em uma discussão entre os tradutores e os pesquisadores, a fim de identificar inconsistências, bem como realizar as suas correções.

\section{Análise por uma comissão de especialistas:}

A primeira versão da escala dos pacientes foi analisada por uma comissão de especialistas, composta por um psicólogo e dois psiquiatras que trabalhavam em serviços de saúde mental - que estavam, portanto, familiarizados com a população-alvo - e pelos dois primeiros autores. A comissão teve como objetivo adequar os termos da escala ao contexto brasileiro e avaliar a equivalência semântica dessa versão com a escala original. A partir das modificações sugeridas, elaborou-se uma segunda versão do instrumento. No caso da escala dos familiares, a comissão reuniu-se em um segundo momento, depois de terminada a adaptação completa da escala dos pacientes, para fazer a redação das questões dirigidas aos familiares dos pacientes, a partir das questões da escala dos pacientes.

5. Estudo piloto: No estudo piloto, a escala foi aplicada a um grupo de usuários, visando testar sua aplicabilidade e facilidade de compreensão pela população-alvo. A versão dos pacientes foi aplicada a 23 pacientes psiquiátricos que frequentavam o serviço e a versão dos familiares, a 20 familiares de pacientes.

A aplicação das escalas foi feita oralmente; as questões foram lidas e as respostas, anotadas pelos entrevistadores. Para verificar a compreensão das palavras usadas na formulação das questões da escala, foi utilizada a técnica de sondagem (Probe Technique), descrita por Guillemin et al. ${ }^{20}$. Segundo esse procedimento, pedia-se aos participantes que justificassem, uma a uma, as suas respostas a cada item da escala. A partir dessa explicação, eram identificados os termos mal compreendidos pelos participantes e procurava-se substituí-los por palavras mais simples e adequadas à população-alvo. Buscava-se, ainda, identificar, nas explicações fornecidas por eles, situações culturalmente pertinentes que pudessem auxiliar no fornecimento de exemplos para os itens da escala. Esses termos, assim identificados na entrevista, eram, então, incorporados aos itens da escala, com reformulações das questões. A escala, assim reformulada, era reaplicada na entrevista seguinte, seguindo-se o mesmo procedimento, até que não fossem encontrados mais problemas de compreensão dos termos.

\section{RESULTADOS}

O processo de adaptação transcultural resultou em algumas modificações na escala EMP-pacientes. A primeira modificação realizada foi a sua redação em forma de entrevista, e não de autoaplicação, como era o formato da escala ori- ginal. Essa modificação permitiria sua aplicação a sujeitos com baixo nível de escolaridade, ou mesmo analfabetos, o que é mais adequado à população-alvo dos serviços de saúde mental, no contexto brasileiro.

A segunda modificação foi feita após o consenso da comissão de especialistas, em concordância com um dos autores da escala original, sobre o número de alternativas de resposta para os itens da escala, que deveria ser diminuído para se obter equilíbrio ou balanceamento na distribuição dos seus valores. Na escala original, são quatro as alternativas de resposta dos itens: uma negativa, uma neutra e duas positivas, a saber: $1=$ pior do que antes, $2=$ sem mudança; 3 = um pouco melhor do que antes, $4=$ muito melhor do que antes. Esse formato apresenta desequilíbrio entre a pontuação negativa e a positiva, ou seja, existem duas alternativas que remetem à melhora e apenas uma que remete à piora, o que não é ideal para um instrumento de medi$\mathrm{da}^{21}$. Na versão brasileira da escala, o número de alternativas foi balanceado, de modo a se obter o mesmo número de alternativas, no polo positivo e no polo negativo do contínuo de avaliação, totalizando três: 1 = melhor do que antes, 2 = sem mudança e 3 = pior do que antes. Outra justificativa para tal diminuição é que ela favorece a compreensão das alternativas de resposta, que se tornam mais fáceis à medida que seu número diminui.

A terceira modificação foi a inclusão de exemplos, colocados entre parênteses, em cinco itens da escala, para que o sentido da questão ficasse mais concreto e fácil de entender, evitando que ela pudesse ter mais de uma interpretação. Por exemplo, no item 8, que avalia a percepção de possíveis mudanças ocorridas na "energia" do paciente, exemplificou-se energia como sendo "disposição para fazer as coisas". No item 17, referente a "tarefas de casa", acrescentou-se um exemplo adicional entre os que já estavam incluídos nesse item. Os exemplos já incluídos na escala original eram cozinhar, lavar roupas e fazer compras no supermercado, o que no contexto brasileiro poderia ser visto como tarefas tipicamente femininas. Decidiu-se então, na comissão de especialistas, incluir um outro exemplo, que foi "consertar coisas na casa".

A quarta modificação refere-se à reformulação da redação de alguns itens da escala, com o objetivo de obter uma linguagem mais simples e próxima do cotidiano, aumentando sua clareza e facilitando sua compreensão por parte dos respondentes. Essas modificações foram testadas durante as entrevistas com os usuários, no estudo piloto. Ao todo, nove itens foram modificados para essa finalidade. Por exemplo, nos itens 12, 13 e 14, ao se avaliarem as mudanças ocorridas nas relações do paciente com pessoas de sua família, amigos e conhecidos, o termo "relações" foi substituído por "convivência", uma vez que o termo relações estava produzindo conotações e entendimentos ambíguos e a palavra convivência mostrou-se mais adequada. 
Foi retirado da escala um dos itens que se referia à situação financeira do paciente, uma vez que a pergunta carecia de sentido no caso da população-alvo estudada. Segundo um dos autores da escala original, esse item se refere especificamente a pacientes usuários de drogas, cuja situação financeira se deteriora por causa da dependência química, em virtude dos gastos constantes com a compra de drogas.

Finalmente, foram acrescentadas duas questões abertas ao final da escala, com o objetivo de colher informações adicionais que pudessem contribuir para a melhoria do tratamento no serviço. Foram as seguintes: "O que piorou em sua vida desde o início do tratamento?" e "Você acha que o tratamento que você recebe aqui pode ser melhorado? Se sim, como? Se não, por quê?".

No que se refere à versão dos familiares, os itens foram elaborados com base na versão dos pacientes, previamente testada. Na redação dos itens, foram modificados os pronomes em todas as questões, visando adequar a escala à população-alvo de familiares. Os resultados do estudo piloto mostraram que a redação dos itens estava clara e de fácil compreensão para os familiares entrevistados, não tendo sido necessário modificar mais a redação de nenhum dos itens. Esse resultado se deve, provavelmente, ao fato de a versão dos pacientes ter sido detalhadamente analisada e cuidadosamente reformulada anteriormente, a partir do estudo piloto feito com os pacientes, não deixando dúvidas quanto à aplicabilidade e à facilidade de compreensão dos itens. Além disso, a população-alvo dos familiares apresentou, em geral, maior facilidade de contato e de compreensão.

\section{DISCUSSÃO}

Este estudo possibilitou realizar a adaptação transcultural da escala canadense de percepção de mudança pelos pacientes como resultado do tratamento em serviços de saúde mental e elaborar, a partir dela, uma escala dirigida aos familiares sobre as mudanças percebidas por eles na vida do paciente. Os instrumentos têm se mostrado de fácil compreensão e aceitação por parte dos pacientes e dos familiares.

No processo de adaptação e construção dos instrumentos, foi de grande importância a discussão com os grupos de especialistas e, particularmente, a realização do estudo piloto. A participação dos especialistas foi essencial para a reformulação das questões e das alternativas de resposta, para inclusão de exemplos em alguns itens e para a verificação da equivalência semântica da escala dos pacientes com a escala original. O estudo piloto possibilitou a escolha de termos mais simples e de fácil compreensão e evidenciou a importância da inclusão de exemplos na escala. Esses procedimentos permitiram testar a validade de conteúdo das questões formuladas para as duas versões da escala EMP22. Procedimentos de adaptação transcultural de instrumentos de medida podem variar entre os pesquisadores, alguns sugerindo a utilização de dois tradutores independentes, tanto para a fase de tradução quanto para a de retradução do instrumento ${ }^{23}$. No presente trabalho, não foi adotado esse procedimento, porém as demais etapas realizadas possibilitaram contornar essa limitação, por exemplo, a comparação e a discussão das duas versões da escala, traduzida e retraduzida, pelos tradutores e o pesquisador, a discussão realizada com a comissão de especialistas e a realização do estudo piloto, utilizando-se a técnica da sondagem, na qual a compreensão dos itens é detalhadamente analisada e reformulações são incorporadas na redação das questões.

As escalas EMP, nas suas duas versões, do paciente e do familiar, poderão ser úteis para estimular a prática de avaliação de resultados, no contexto brasileiro, com um instrumento simples, curto e de fácil aplicação. Essa prática pode contribuir para a melhoria da qualidade dos serviços de saúde mental quando as informações colhidas são incorporadas ao tratamento9. Além disso, a utilização de instrumentos de medida que tenham sido submetidos aos procedimentos de adaptação e validação recomendados internacionalmente permite aumentar a validade e a confiabilidade dos dados e a comparabilidade dos resultados obtidos por pesquisas realizadas em diferentes serviços e contextos, permitindo assim o acúmulo de conhecimento na área, ${ }^{911}$.

Avaliar se o tratamento realizado nos serviços produz mudanças na vida dos pacientes, detectadas por eles próprios e por seus familiares, fornece também uma medida de validação social dos resultados do tratamento. Serve, portanto, de calibração das medidas objetivas, pois permite verificar se mudanças estatisticamente significativas detectadas pelos profissionais produzem impacto real na vida dos pacientes, uma vez que nem sempre a significância estatística se acompanha de significância clínica social³.

Deve-se considerar, entretanto, que a avaliação dos resultados pelos próprios pacientes constitui uma medida complementar, que deve ser tomada com as demais aferições objetivas dos efeitos do tratamento, e não ser considerada como uma medida substitutiva"1" Vieses típicos de medidas de autorrelato podem ocorrer, tais como efeitos de "desejabilidade social" e racionalização, devendo ser levados em consideração na interpretação dos resultados. No caso específico de serviços de saúde, os pacientes podem apresentar informações distorcidas, por causa de fatores como baixa expectativa, falta de assertividade, excesso de tolerância, desvio de atenção de alguns aspectos por outros mais visíveis no serviço, entre outros ${ }^{7}$. Podem ocorrer vieses que resultam em respostas excessivamente positivas, como nas medidas de satisfação, tais como o viés de gratidão e/ou de aquiescência e, no caso de serviços públicos, por serem gratuitos, o receio de perder o acesso ao serviço ${ }^{24}$. Cuidados metodológicos devem ser tomados para diminuir a probabilidade desses vieses no contexto de uma pesquisa, como o uso de instruções de- 
talhadas, destacando a importância de uma informação válida e a garantia de anonimato ${ }^{3,9}$. Cuidados referentes à privacidade durante as entrevistas de coleta de dados e a garantia de que as informações não resultarão em perda de benefícios sociais podem ser importantes, pois alguns pacientes ou familiares receiam que suas respostas possam prejudicá-los, por exemplo, resultando na perda de um benefício ou pensão. O procedimento de sondagem adotado nas entrevistas desta pesquisa, no qual era solicitada uma explicação para a resposta dada a cada item da escala, pode igualmente contribuir para a segurança dos dados.

Tomadas as devidas precauções, pesquisas utilizando as escalas de mudança percebida poderão fornecer informações importantes sobre os resultados do tratamento, que podem contribuir para a melhoria dos serviços de saúde mental no contexto brasileiro. Essas medidas podem ser usadas, ainda, para investigar as relações entre a ocorrência de melhora percebida pelos usuários e características do tratamento recebido. Segundo Hansson? ${ }^{9}$, há necessidade de se promover pesquisas que investiguem quais são os fatores preditivos de resultados positivos do tratamento, buscando conhecer as relações entre esses resultados e variáveis como as características do serviço ou das intervenções utilizadas no tratamento do paciente, para aumentar o conhecimento sobre quais aspectos do tratamento são mais efetivos.

\section{CONCLUSÃO}

As escalas EMP são de fácil compreensão pelos usuários, independentemente do nível socioeconômico deles, e apresentam equivalência semântica com a escala original. Elas poderão ser utilizadas para avaliar os efeitos do tratamento em serviços de saúde mental, o impacto de programas ou intervenções terapêuticas específicas e os resultados de um tratamento medicamentoso, nas diversas dimensões da vida dos pacientes. As escalas permitem colher informações que poderão redirecionar as prioridades do atendimento, pois, além de indicar em quais dimensões está ocorrendo melhora, também apontam para as dimensões em que não está ocorrendo melhora e as que chegaram mesmo a piorar, necessitando, portanto, de atenção especial e mudança nas intervenções. Além disso, essas escalas podem ser usadas em pesquisas de levantamento, com amostras mais amplas, por serem curtas, de fácil aplicação e baixo custo. As questões abertas da escala podem indicar, ainda, algum aspecto adicional das necessidades dos pacientes que não foi detectado pelos itens da escala, o que tem se mostrado útil em pesquisas, por desvendar aspectos relativos a demandas dos pacientes ${ }^{25}$.

O estudo das propriedades psicométricas das duas versões da escala EMP, em termos de sua fidedignidade e validade, está sendo realizado atualmente e será objeto de fu- tura publicação. Essas escalas serão disponibilizadas no site do Laboratório de Pesquisa em Saúde Mental da UFSJ, após o estudo e a apresentação de suas qualidades de medidas.

\section{AGRADECIMENTO}

À Fundação de Amparo à Pesquisa do Estado de Minas Gerais (Fapemig) e ao Conselho Nacional de Desenvolvimento Científico e Tecnológico (CNPq), que financiam o projeto de pesquisa do qual este artigo faz parte.

\section{REFERÊNCIAS}

1. World Health Organization (WHO). The World Health Report, Mental Health: New Understanding, New Hope, 2001.

2. Donabedian A. Evaluating the quality of medical care. The Milbank Quarterly. 2005; 83(4):691-729. Milbank Memorial Fund. Published by Blackwell Publishing. Reprinted from The Milbank Memorial Fund Quarterly. 1966;44(3):166-203.

3. Mercier L, Landry M, Corbiere M, Perreault M. Measuring clients' perception as outcome measurement. In: Roberts AR, Yeager KR. Evidence-based Practice Manual: Research an Outcome Measures in Health and Human Services. Oxford: University Press; 2004. p. 904-9.

4. FDA Guidance for industry - Patient-reported outcome measures: use in medical product development to support labeling claims. US Department of Health and Human Services, Food and Drug Administration, Center for Drug Evaluation and Research (CDER), Center for Biologics Evaluation and Research (CBER), Center for Devices and Radiological Health (CDRH). Clinical/Medical; February, 2006.

5. McCabe R, Saidi M, Priebe S. Patient reported-outcomes in Schizophrenia. Br J Psychiatry. 2007;191(Suppl 50):21-8.

6. Fadhoury WKH, Kaiser W, Roeder-Wanner U, Priebe S. Subjective evaluation: Is there more than one criterion? Schizophr Bull. 2002;28(2):319-27.

7. Donabedian A. Quality assurance in health care: consumers' role. Quality in Health Care. 1992;: $247-51$.

8. Holcomb WR, Parker JC, Leong GB, Thiele J, Higdon J. Customer satisfaction and selfreported treatment outcomes among psychiatric inpatients. American Psychiatric Association. Psychiatr Serv. 1998;49:929-34.

9. Hansson L. Outcome assessment in psychiatric service evaluation. Soc Psychiatry Psychiatr Epidemiol. 2001;36:244-8.

10. Hasler G, Moergeli H, Bachman R, Lambreva E, Buddebeg C, Schnyder U. Patient satisfaction with outpatient psychiatric treatment: the role of diagnosis, pharmacotherapy and perceived therapeutic change. Can J Psychiatry. 2004:49:315-21.

11. Ruggeri AE. Patients' and relatives' satisfaction with psychiatric services: the state of the art of its measurement. Soc Psychiatr Epidemiol. 1994;29:212-27.

12. Priebe S, Gruyters T. Patients' assessment of treatments predicting outcome. Schizophr Bull. 1995;21(1):87-94.

13. Ribeiro MS, Alves MJM, Vieira EMM, Silva PM, Lamas CVDB. Fatores associados ao abandono de tratamento em saúde mental em uma unidade de nível secundário do Sistema Municipal de Saúde. J Bras Psiquiatr. 2008;57(1):16-22.

14. Tait L, Lester $H$. Encouraging user involvement in mental health services. Adv Psychiatr Treat. 2005;11:168-75.

15. Bandeira M, Barroso SM. Sobrecarga das famílias de pacientes psiquiátricos. J Bras Psiquiatr. 2005;54(1):34-46.

16. Tessler RC, Gamache GM. Family experiences with mental illness. Westport: Auburn House; 2000.

17. Bandeira M, Pitta AMF, Mercier C. Escalas da OMS de avaliação da satisfação e da sobrecarga em serviços de saúde mental: qualidades psicométricas da versão brasileira. J Bras Psiquiatr. 1999;48(6):233-44. 
18. Bandeira M, Pitta AMF, Mercier C. Escalas de avaliação da satisfação dos usuários em serviços de saúde mental: SATIS-BR. J Bras Psiquiatr. 2000;48(8):293-300.

19. Vallerand RJ. Vers une méthodologie de validation trans-culturelle de questionnaires psychologiques: implications pour la recherché en langue française. Canadian Psychology. 1989;30(4):662-80.

20. Guillemin F, Bombardier C, Beaton D. Cross-Cultural adaptation of health-related quality of life measures: literature review and proposed guidelines. J Clin Epidemiol. 1993; 46(12):1417-32

21. Gunther H. Como elaborar um questionário. In: Pasquali L, editor. Instrumentos Psicológicos: Manual Prático de Elaboração. Brasília: LabPAM, IBAPP; 1999. p. 231-58.
22. Fachel JMG, Camey S. Avaliação psicométrica: a qualidade das medidas e 0 entendimento dos dados. In: Cunha JA, organizador. Psicodiagnóstico. 5a ed. Porto Alegre: Artmed; 2000. p. $158-70$.

23. Moraes $C L$, Hasselman MH, Reichenheim ME. Adaptação transcultural para o português do instrumento "Revised Conflict Tactics Scales (CTS2)" utilizado para identificar violência entre casais. Cad Saúde Pública. 2002;18(1):163-76.

24. Esperidião M, Trad LAB. Avaliação da satisfação de usuários. Ciência e Saúde Coletiva. 2005;10(Sup):303-12.

25. Tempier R, Pawliuk N, Perreault M, Steiner W. Satisfaction with clinical case management services of patients with long-term psychoses. Community Ment Health J. 2002;38(1):51-9. 